\title{
Valoración de los contenidos de Expresión Corporal por parte de los futuros maestros en la asignatura de Actividades Físicas Artístico-expresivas de la mención de Educación Física \\ Assessment of the contents of Body Expression by future teachers in the subject of Artistic-expressive Physical Activities of the mention of Physical Education \\ Jorge Carlos Lafuente \\ Universidad de Burgos (España)
}

Resumen: La Expresión Corporal se caracteriza por sus carencias en la implementación en el área de Educación Física, entre las causas se encuentran: la falta de formación del profesorado, la difícil impartición de contenidos no habituales, el desconocimiento de éstos o las malas experiencias previas. El presente artículo pretende analizar los diferentes contenidos llevados a cabo en la asignatura de Actividades Físicas Artístico-Expresivas, de la Mención de Educación Física del Grado de Magisterio en la Universidad de Burgos. Se quiere conocer qué contenidos son considerados más educativos, cuáles resultan más difíciles de impartir en un aula de Primaria y cuáles han generado más experiencias positivas. Se utiliza una metodología mixta a través de la realización de un cuestionario ad hoc y el análisis de los diarios del alumnado. Tras el análisis de los resultados, se observa que los estudiantes valoran en general los contenidos como muy educativos, sin excesiva dificultad y generadores de experiencias positivas. Aspectos como la necesidad de material, el género, la capacidad de evitar bloqueos y mejorar la confianza del alumnado son claves para la valoración de los contenidos.

Palabras claves: Expresión Corporal, formación del profesorado, contenidos, Educación Física, Educación Primaria.

\begin{abstract}
Physical Education does not always deal with Body Expression. Among the causes for this are lack of teacher training, difficult delivery of unusual content, ignorance or previous bad experiences. This article aims to analyze the different topics included in the subject of Artistic-Expressive Physical Activities in the Mention of Physical Education Teaching Degree in the University of Burgos. It intends to show which contents are more educational, which are more difficult to teach in Primary School and which have generated more positive experiences. A mixed methodology is used with an ad hoc questionnaire and the analysis of student diaries. After analysis of the results, it is observed that students generally value these contents as very educational, they are not excessively difficult and generate positive experiences. Some key aspects for the assessment of the content are the ability to avoid blockage and improve student confidence, gender and the need for materials.
\end{abstract}

Key words: Body Expression, teacher training, content, Physical Education, Primary Education.

\section{Introducción}

El desarrollo de la autoestima, la generación de relaciones interpersonales o la conexión con otras áreas del currículum y aprendizajes escolares son entre otras, algunas de las fortalezas de la Expresión Corporal dentro del contexto de la Educación Física (LLuch, Urzúa, León-Prados, \& Sánchez, 2011). Por otro lado, todos los bloques de contenido entre ellos el relativo a la Expresión Corporal, que se proponen en la Ley educativa (Ley orgánica 8/2013) deben desarrollarse en su plenitud, para conseguir que la Educación Física pueda contribuir al desarrollo integral del sujeto (Rodríguez, Robles, Viera, Fuentes-Guerra, \& Rodríguez, 2013). En este

Fecha recepción: 29-03-21. Fecha de aceptación: 16-06-21

Jorge Carlos Lafuente Fernández

gitocarlos@hotmail.com sentido, se debe equiparar la Expresión Corporal a los contenidos conocidos como tradicionales, deportes y condición Física (Aijón, 2012), debido a que en muchas ocasiones queda marginada, trabajándose solo en momentos determinados, como realizar una coreografía o baile para navidad o fin de curso (Megías 2020) Todo esto, hace que la Expresión Corporal deba formar parte de las programaciones de los profesores de Educación Física y sea parte importante en la formación universitaria de futuros docentes.

A pesar de lo expuesto, la Expresión Corporal no se trabaja en toda su amplitud dentro de la Educación Física (Rodríguez et al., 2013). Existen varios problemas que pueden hacer que esto suceda. Algunos están relacionados con el contenido, con el alumnado y con el docente. En relación con el contenido, la Expresión Corporal ha sido considerada durante años como un cajón de sastre (García \& Gutiérrez, 2002; Sánchez \& 
López, 2019) donde se han incluido contenidos muy diversos. García \& Gutiérrez (2002) hablan de la gran amplitud de contenidos dentro de la Expresión Corporal y su continuo crecimiento, haciendo todavía más complicado su delimitación, no existiendo un acuerdo entre los distintos profesionales sobre los contenidos propios de Expresión Corporal (García \& Gutiérrez, 2002; Gil, 2016). Debido a esto, es necesario conocer qué contenidos, los futuros docentes, ven como más educativos o difíciles de llevar a cabo, ya que podría ser el principio de una línea de trabajo común.

El futuro docente es clave en el desarrollo de la Expresión Corporal, ya que si un docente ve un contenido con pocas posibilidades educativas u observa que es un contenido difícil de llevar a cabo en el aula de Primaria, podría optar por darle menor importancia, dedicarle menos tiempo o directamente obviarlo. Además, el profesor es clave a la hora de mejorar las actitudes y la motivación del alumnado hacia la Expresión Corporal (Arias, Fernández \& San Emeterio (2020); Arias, Fernández \& Valdés, 2021; Bores-García et al. 2021). Para conseguir esto es fundamental incidir en una buena formación de Expresión Corporal para los futuros maestros. Sin embargo, es habitual que se hable de un déficit en la formación de contenidos de Expresión Corporal (Archilla Prat, 2013; García \& Gutiérrez, 2002), presentándose esta habitualmente, con una carga de créditos reducida y solo durante un año (Prat \& Brunicardi, 2012), además de una falta de claridad en la formación inicial, con una carencia de información estructurada y unificadora (García \& Gutiérrez, 2002). Este problema, hace necesaria la valoraciónn o análisis de aspectos importantes en la formación universitaria de la Expresión Corporal.

La formación de los futuros maestros, debe ser algo más que la mera impartición de contenidos, se debe orientar la enseñanza a la adquisición de recursos, la construcción de la identidad profesional y al resurgimiento de la labor vocacional del maestro (Köpsén, 2014). De esta manera, es importante que el alumnado entre otras muchas tareas, reflexione sobre los contenidos que reciben y analicen si estos son educativos, si tienen posibilidades reales de llevarse a cabo, si les han parecido interesantes o si les gustan, para poder ir construyendo así su identidad profesional.

Erickson (2004) afirma que un método eficaz para involucrar a los alumnos es presentarles temas relacionados con sus intereses y experiencias. Esta misma idea se observa al tratar la formación académica en Expresión Corporal, así Ares y López (2012) plantean que, cuando los contenidos que se imparten son considerados relevantes para los futuros docentes, se aumentan las posibilidades de que estos contenidos se lleven a cabo más adelante. Por lo tanto, para conseguir que los futuros docentes lleven a cabo unidades didácticas de Expresión Corporal en el aula, es interesante guiar los contenidos impartidos en su formación, hacia aquellos más relevantes para ellos. En este sentido Llopis y García (2012) estudiaron las experiencias sobre danza que tenían los estudiantes de la Licenciatura de Ciencias de la Actividad Física y el deporte y de la diplomatura de Educación Física , encontrando que los estudiantes apenas habían realizado actividades de danza y la mitad de estos no habían trabajado la Expresión Corporal. Señalando que los estudiantes que más valoraban estos contenidos, eran los que anteriormente habían realizado experiencias de danza y expresión corporal. Teniendo en cuenta esto Arias, Fernández y San Emeterio (2020) señalan que el no impartir o impartir la expresión corporal con desconocimiento influye en las actitudes negativas de los alumnos hacia la expresión corporal. Al hablar de contenidos, el RD 126/2014 define estos como: «conjunto de conocimientos, habilidades, destrezas y actitudes que contribuyen al logro de los objetivos de cada enseñanza y etapa educativa y a la adquisición de competencias.» (p. 5), por tanto, en el contexto escolar, un contenido educativo debería cumplir esta definición. En el ámbito Universitario, Ares y López (2012) preguntaron a los alumnos del Grado de Ciencias del Deporte sobre los contenidos de Expresión Corporal. Estos valoraron positivamente los contenidos propuestos considerándolos relevantes para su formación y su futuro profesional, consiguiendo de esta manera contribuir al logro de los objetivos propuestos en el contexto universitario, cumpliendo así con el aspecto educativo de esos contenidos.

Dentro de la Expresión Corporal, se podrían utilizar muchos y muy diferentes contenidos que cumplan los requisitos expresados en la Ley, poniendo así más difícil tal y como se ha expuesto anteriormente, un acuerdo entre los autores (García \& Gutiérrez, 2002; Gil, 2016). Un intento de analizar los contenidos en Expresión Corporal es el realizado por Sánchez y López (2019) revisando la formación inicial de los docentes de Primaria en Expresión Corporal y contrastando los contenidos propuestos en las guías docentes de las universidades públicas españolas. Para este análisis establecen cinco categorías de contenidos: Toma de conciencia de uno mismo, comunicación, creatividad, disciplinas artísticas y otros contenidos. Tras examinar los documentos, se observa 
como gran parte de las guías contienen elementos pertenecientes a las categorías comunicaciónn y disciplinas artísticas; y como la práctica es prioritaria con respecto a la teoría. A pesar de la importancia que tienen en concreto las disciplinas artísticas, Sánchez y López (2019) comentan que hay que tener en cuenta que la Expresión Corporal tiene una técnica que debe servir para profundizar en la toma de conciencia del cuerpo y de uno mismo para llegar a la comunicación y la expresiónn creativa a través del movimiento. Estando en contra de aprendizaje por imitación o copia de técnicas de otras disciplinas artísticas. A pesar de esto, las técnicas de estas disciplinas bien utilizadas pueden complementar y enriquecer la formaciónn del docente.

Una de las mayores dificultades al realizar tareas de Expresión Corporal puede surgir cuando el alumno se siente observado, debido a que las tareas con interacción visual, podrían funcionar como inhibidor del compromiso emocional del alumnado (Arizcuren, 2013; Canales-Lacruz \& Arizcuren-Blasco, 2019). Canales-Lacruz, Táboas-País y Rey-Cao (2013) examinan las percepciones de los estudiantes en la asignatura de EC perteneciente a la licenciatura en Ciencias de la actividad Física y el deporte, en cuanto a la desinhibición, la espontaneidad y la codificación de tareas en Expresión Corporal, concluyendo como la interacción visual y táctil inhibe el compromiso expresivo, por lo que construir espacios de seguridad sería necesario (Meirieu, 1988). En esta línea Torrents et al. (2011) a partir de las emociones manifestadas también por alumnos de Expresión Corporal en la licenciatura en ciencias de la actividad Física y el deporte, observa como el alumnado manifiesta sentir vergüenza cuando se expresa con el cuerpo fundamentalmente en su iniciación. A pesar de esto, en general, los estudiantes tienen emociones positivas, generadas probablemente por experiencias positivas. Canales-Lacruz y Corral-Abós (2021) observan también como las tareas de Expresión Corporal, en la asignatura de la mención de Educación Física del grado de maestro de primaria, generan más comodidad que incomodidad en el alumnado, condicionando la interacción visual y el compromiso emocional de estos.

La principal contribución del estudio es el análisis exhaustivo de los contenidos impartidos en la formación en Expresión Corporal de los futuros docentes. La investigación tiene un objetivo principal, evaluar los contenidos de Expresión Corporal impartidos a los futuros docentes de Educaciónn Física y tres objetivos específicos. Evaluar qué contenidos para los futuros maestros: a) son más educativos; b) cuáles resultan máss difíciles de impartir en un aula de Primaria; c) cuáles han generado más experiencias positivas a los alumnos de la asignatura de Expresión corporal.

\section{Material y método}

\section{Participantes}

La muestra está formada por todos los alumnos que cursan la asignatura de Actividades Físicas Artístico-Expresivas por la modalidad presencial en la mención de Educaciónn Física del Grado en Educación Primaria de la Universidad de Burgos, 18 sujetos. De estos 6 chicas y 12 chicos. La media de edad fue de $23.6 \pm 5.5$ años.

\section{Instrumentos}

\section{Cuestionario}

Para la recogida de la información se elabora un cuestionario ad hoc, organizado en tres bloques, en función de los tres objetivos específicos de la investigaciónn. El instrumento lo forman 24 preguntas, ocho preguntas por cada bloque con ítems de escala de construcción tipo Likert con 5 alternativas (de 1-nada a 5-mucho). Las ocho preguntas, se corresponden con los ocho contenidos estudiado:

Bloque I. Información relativa a lo educativo que consideran los alumnos/as de Magisterio el contenido tratado en cada sesiónn práctica. (Preguntas $1 a, 1 b, 1 c, 1 d, 1 e$, If, $1 \mathrm{~g}, 1 \mathrm{~h})$. Bloque II. Dificultad percibida por los alumnos/as de Magisterio para impartir en un aula de Pri-

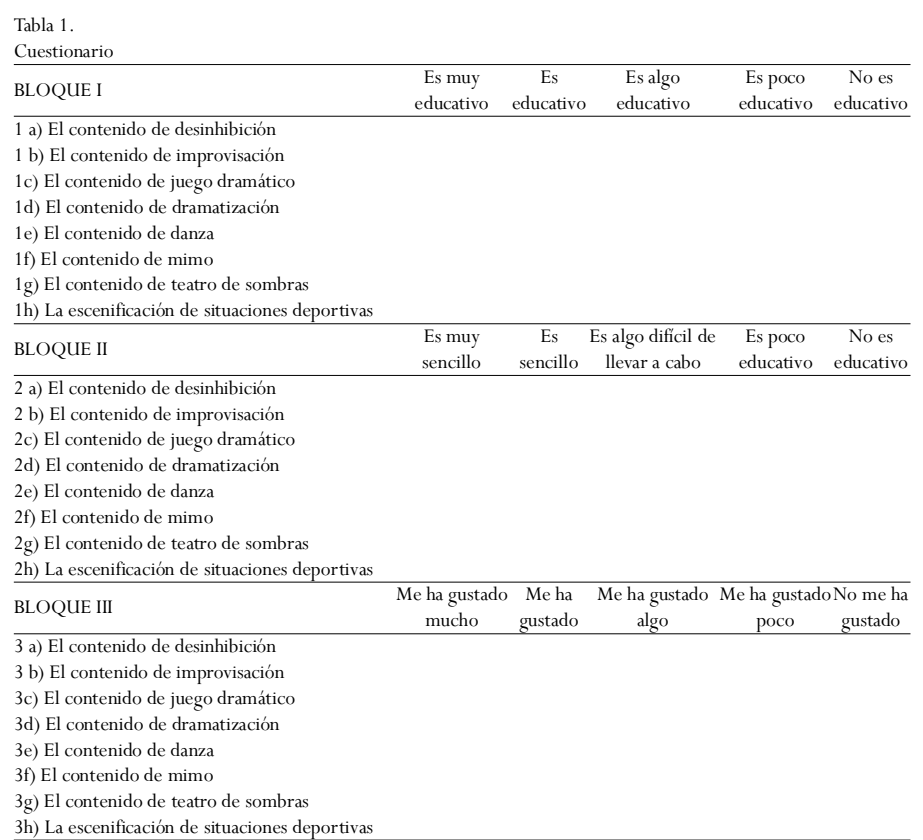


maria el contenido tratado. (Preguntas 2a, 2b, 2c, 2d, 2e, 2f, $2 \mathrm{~g}, 2 \mathrm{~h}, 2 \mathrm{i})$.

Bloque III. Información referente a si los contenidos impartidos han generado experiencias positivas en los alumnos/as de Magisterio. (Preguntas 3a, 3b, 3c, 3d, 3e, 3f, 3g, 3h, 3i).

\section{Diario del alumno}

El alumno después de cada sesión práctica, registra los aspectos más relevantes acontecidos relativos a los ejes de estudio. Este diario se rige por una estructura previa facilitada por el docente, en este se sugieren tres preguntas, que invitan a la reflexión a través de una respuesta abierta.

Tabla 2.

Guion básico para la realización de los diarios del alumnado.

1. ¿Te ha parecido educativo el contenido tratado hoy en clase?

2. Te ha parecido sencillo de llevar a cabo en un aula de Primaria?

3. ¿Te ha gustado la sesión?

\section{Diseño y procedimiento}

La investigación se estructuró en tres fases.

Fase 1. Diseño del estudio y contacto con participantes: Primero se delimitó los objetivos del estudio, la metodología a realizar y se diseñó el diario de sesiones de los alumnos y el cuestionario ad hoc. Con relación a los estudiantes, debido a que el investigador impartía docencia en la asignatura AFAE, se les explicó en la primera sesión de la asignatura como debían realizar el diario de sesiones y cuando se les pasaría el cuestionario. En esta investigación el profesor adopta un enfoque crítico hacia el curriculum y la enseñanza, adoptando una dirección investigadora. El profesor reflexiona, discute y cuestiona su propia práctica para la mejora de la enseñanza (Elliot, 2000).

Fase 2. Realización de sesiones prácticas, diarios de sesión de los alumnos y cuestionario: desde la asignatura AFAE se seleccionaron ocho contenidos. Estos se impartieron en una sesión teórica y una sesión práctica, de dos horas de duración cada una, agrupados según un objetivo, disciplina artística o elemento común a través de juegos, técnicas, tareas, ejercicios, dinámicas, propuestas y todo tipo de recursos de la Expresión Corporal propuestos en la guía docente. El orden de impartición de estos fue el siguiente: $1^{\circ}$ juegos de desinhibición, $2^{\circ}$ juegos de improvisación, $3^{\circ}$ juegos dramáticos, $4^{\circ}$ dramatización $5^{\circ}$ danza, $6^{\circ}$ mimo, $7^{\circ}$ teatro de sombras y $8^{\circ}$ escenificación de situaciones deportivas. Las clases teóricas consistían en dos horas dedicadas a cada contenido. En estas se desarrolló conceptos teóricos, fases, pro- puestas prácticas, progresiones, ... de cada uno, a través del uso de power point y videos. Además de estas, en las sesiones prácticas se impartían al alumnado juegos y unidades didácticas, relacionadas con el contenido a trabajar. Los estudiantes realizaban estas actividades, pero también proponían e impartían algunas, al resto del alumnado. Al final de cada sesión práctica, se llevó a cabo una puesta en común donde los alumnos realizaron una evaluación conjunta del contenido presentado. Más tarde, de forma individual cada alumno completó su diario, según el planteamiento dado por el profesor. Una vez impartidas todas las sesiones teóricas y prácticas se le pasó al alumnado el cuestionario.

Fase 3. Categorización de la información y análisis en profundidad de los resultados: Esta fase tuvo dos partes, por un lado, con los datos de los cuestionarios se realizó un análisis descriptivo planteado en torno a tres categorías. Por otro lado, la información recogida de los diarios de los alumnos fue analizada bajo criterios de saturación de la información, generando también tres categorías para el análisis de resultados.

\section{Análisis de datos}

Se ha realizado una metodología mixta trabajando con datos cuantitativos y cualitativos, considerando que ambas metodologías se complementan (Sánchez, 2015, p.17).

Por un lado, se ha utilizado una metodología de corte cualitativo, realizando el análisis de contenido a través de la revisión de la información recogida en el diario del alumno, utilizando patrones cruzados coincidentes, para favorecer la codificación y contabilización de los extractos de texto generados (Saldaña, 2009). Se trata de un estudio exploratorio que indaga en la valoración de los contenidos de Expresión Corporal por parte de los futuros de Educación Física . Los resultados se organizaron en tres categorías, según un proceso de codificación abierto, axial y selectivo. Se utilizó la saturación como premisa previa para cribar la información, lo que favoreció la contabilización de los extractos de texto y la presentación de aquellos más significativos.

Por otro lado, se ha realizado un análisis cuantitativo y descriptivo de los datos del cuestionario a través del paquete estadístico SPSS versión 20.0. En este se han extraído las medidas de tendencia central y dispersión. Este análisis cuantitativo pretende complementar la metodología cualitativa, para exponer las opiniones de los alumnos del cuestionario final y contrastarlas con aquellas que fueron reflejando previamente en el diario. 
Generación de categorías y su categorización

Se han generado tres categorías para estructurar los resultados, estas subyacen de la temática del estudio y de los objetivos de la investigación. La información de cada una de las categorías se relaciona directamente con las preguntas que se han establecido en el diario de los alumnos, garantizando así la objetividad y la linealidad de la investigación (Oliver-Hoyo \& Allen, 2006). Además, cada una de las categorías recoge información vinculada a diversas temáticas, las cuales se han estructurado en subtemas dentro de los resultados. Esta estructuración favorece la triangulación y la saturación de los datos obtenidos (Tortorella, Viana \& Fettermann, 2015) Estas categorías son:

Contenidos educativos en Expresión Corporal: se analiza todo lo referente a las potencialidades de los contenidos impartidos, los ámbitos que desarrollan en el alumnado, la importancia de desarrollar estos en Primaria y aspectos de EC que trabajan.

Grado de dificultad en la impartición de los contenidos de Expresión Corporal en el aula de Primaria: se analizan aspectos vinculados a la necesidad de material o instalación específica. Rechazo o motivación del alumnado hacia los contenidos. La sencillez y la progresión de los ejercicios o tareas.

Experiencias positivas generadas por los contenidos de EXpresión Corporal. Se analizan aspectos relativos a las sensaciones o sentimientos generados durante la práctica. Las experiencias previas y los comportamientos y actitudes observados en ellos y en sus compañeros.

\section{Resultados}

\section{Análisis del cuestionario}

A continuación, se exponen los principales resultados obtenidos en relación al primer bloque del cuestionario sobre la valoración de lo educativo de los contenidos impartidos. En la tabla 3 se observa como en general los contenidos son valorados como bastante educativos, obteniendo la gran mayoría una media mayor de 4 y no habiendo gran diferencia entre ellos. El contenido más educativo para el alumnado son los juegos de desinhibición (media 4.38) considerándolos la mitad de la clase muy educativos, seguidos de la dramatización

\begin{tabular}{|c|c|c|c|c|c|c|c|}
\hline & media & DT & $\begin{array}{c}\text { No es } \\
\text { educativo }\end{array}$ & $\begin{array}{c}\text { Poco } \\
\text { educativo }\end{array}$ & $\begin{array}{c}\text { Algo } \\
\text { educativo }\end{array}$ & Educativo & $\begin{array}{c}\text { Muy } \\
\text { educativo }\end{array}$ \\
\hline Desinhibición & 4.38 & 0.69 & - & - & 11.1 & 38.9 & 50 \\
\hline Improvisación & 4.22 & 0.64 & & - & 11.1 & 55.6 & 33.3 \\
\hline Juego Dramático & 4.22 & 0.87 & & 5.6 & 11.1 & 38.9 & 44.4 \\
\hline Dramatización & 4.27 & 0.66 & - & & 11.1 & 50 & 38.9 \\
\hline Danza & 4.11 & 0.58 & - & - & 11.1 & 66.7 & 22.2 \\
\hline Mimo & 3.94 & 0.72 & & - & 27.8 & 50 & 22.2 \\
\hline Teatro de sombras & 4.22 & 0.73 & & - & 16.7 & 44.4 & 38.9 \\
\hline
\end{tabular}

(media 4.27), mientras que los juegos de improvisación, el juego dramático y el teatro de sombras tienen la misma media (4.22). En el lado opuesto encontramos la escenificación de situaciones deportivas (media 6.61), seguido de mimo (3.9) y danza (media 4.11).

En el segundo bloque, se preguntaba sobre la valoración en cuanto a la dificultad de llevar a cabo los contenidos en un aula de Primaria. En los datos expuestos en la tabla 4, se observa como para los alumnos el contenido más difícil de llevar a cabo es el teatro de sombras (media 2.94), seguido del mimo (media 3.16) y juegos de improvisación (media 3.22). Por otro lado, la escenificación de situaciones deportivas es considerado el contenido más sencillo (media 3.94), seguido de desinhibición (media 3.55) y juego dramático (media 3.38).

\begin{tabular}{|c|c|c|c|c|c|c|c|}
\hline & media & & $\begin{array}{c}\text { Muy difícil de } \\
\text { llevar a cabo }\end{array}$ & $\begin{array}{c}\text { Difícil de } \\
\text { llevar a cabo }\end{array}$ & & & $\begin{array}{l}\text { Muy } \\
\text { sencillo }\end{array}$ \\
\hline Desinhibición & 3.55 & 0.92 & - & 16.7 & 22.2 & 50 & 11.1 \\
\hline Improvisación & 3.22 & 0.94 & 5.6 & 11.1 & 44.4 & 33.3 & 5.6 \\
\hline Juego Dramático & 3.38 & 0.97 & 5.6 & 11.1 & 27.8 & 50 & 5.6 \\
\hline Dramatización & 3.33 & 1.08 & 11.1 & 5.6 & 27.8 & 50 & 5.6 \\
\hline Danza & 3.27 & 0.66 & & 11.1 & 50.5 & 38.9 & - \\
\hline Mimo & 3.16 & 0.92 & 5.6 & 16.7 & 33.3 & 44.4 & \\
\hline Teatro de sombras & 2.94 & 1.1 & 11.1 & 22.2 & 33.3 & 27.8 & 5.6 \\
\hline $\begin{array}{l}\text { Escenificación de situaciones } \\
\text { deportivas }\end{array}$ & 3.94 & 0.8 & - & 5.6 & 16.7 & 55.6 & 22.2 \\
\hline
\end{tabular}

En relación con la tercera categoría, se pretendía conocer si al alumnado les ha gustado los contenidos impartidos y de esta manera, saber si han tenido una experiencia positiva durante la realización de estos. En la tabla 5, se observa como el teatro de sombras es el contenido con mayor puntuación (media 4.44), en el cual a más de la mitad de la clase $(55,6 \%)$ le ha gustado mucho, este es seguido por los juegos de improvisación (media 4.11) y mimo (media 4.05). En el lado contrario, se encuentra la escenificación de situaciones deportivas (media 3.55), el cual es el contenido que menos ha gustado, seguido de danza (media 3.66) y dramatización (media 3.77). Cabe resaltar el caso de la danza, donde a pesar de que al 50\% de los alumnos les ha gustado y al $16.7 \%$ le ha gustado mucho, también tiene un porcentaje de rechazo mayor que el resto.

Tabla 5

\begin{tabular}{|c|c|c|c|c|c|c|c|}
\hline & media & DT & $\begin{array}{c}\text { No me ha } \\
\text { gustado } \\
\text { nada }\end{array}$ & $\begin{array}{l}\text { Me ha } \\
\text { gustado } \\
\text { poco }\end{array}$ & $\begin{array}{l}\text { Me ha } \\
\text { gustado } \\
\text { algo }\end{array}$ & $\begin{array}{l}\text { Me ha } \\
\text { gustado }\end{array}$ & $\begin{array}{l}\text { Me ha } \\
\text { gustado } \\
\text { mucho }\end{array}$ \\
\hline Desinhibición & 3.94 & 0.72 & - & - & 27.8 & 50.0 & 22.2 \\
\hline Improvisación & 4.11 & 0.67 & - & - & 16.7 & 55.6 & 27.8 \\
\hline Juego Dramático & 3.88 & 0.58 & - & - & 22.2 & 66.7 & 11.1 \\
\hline Dramatización & 3.77 & 0.64 & & - & 33.3 & 55.6 & 11.1 \\
\hline Danza & 3.66 & 1.02 & 5.6 & 5.6 & 22.2 & 50.0 & 16.7 \\
\hline Mimo & 4.05 & 0.87 & & 5.6 & 16.7 & 44.4 & 33.3 \\
\hline Teatro de sombras & 4.44 & 0.70 & & & 11.1 & 33.3 & 55.6 \\
\hline $\begin{array}{l}\text { Escenificación de situaciones } \\
\text { deportivas }\end{array}$ & 3.55 & 1.04 & 5.6 & 5.6 & 33.3 & 38.9 & 16.7 \\
\hline
\end{tabular}




\section{Análisis de los diarios del alumnado (386 ex- tractos de texto)}

Contenidos educativos en Expresión Corporal (146 extractos de texto).

En general el alumnado valora como bastante educativos todos los contenidos, no existiendo ningún comentario que considere poco educativo algún contenido. Al igual que en el cuestionario, el contenido que tiene más comentarios sobre sus posibilidades educativas es la desinhibición. En general los futuros maestros y maestras destacan en sus comentarios de este, la capacidad para disminuir los bloqueos o «vergüenza» y mejorar la confianza del alumnado de Primaria.

"Las actividades realizadas durante esta sesión me parecen educativas ya que enseñan a perder la vergüenza de una forma progresiva haciendo que el alumno inconscientemente empiece a actuar mejorando a su vez, la espontaneidad, la imaginación y el descubrimiento personal» (A.R.). "Gracias a este tipo de juegos y actividades los alumnos son capaces de perder la vergüenza y coger confianza en sí mismos y con los compañeros de forma progresiva» (D.M.). "Creo que los alumnos pierdan la vergüenza de forma recurrente e intencionada es una buena técnica educativa. Sin confianza y autoestima es más difícil hacer trabajos en grupo» (M.A.).

Además, existen comentarios sobre otros contenidos, como por ejemplo en el juego dramático, donde se destaca la importancia de trabajar antes desinhibición.

"Para estos juegos es necesario realizar previamente juegos de desinhibición para que los alumnos pierdan la vergüenza y no les cueste actuar delante de sus compañeros» (D.C.).

La desinhibición además es utilizada para ensalzar el poder educativo de otros contenidos como la danza, donde la música juega un papel importante.

"Creo que el tema de la danza es un contenido muy educativo y poco explotado, ya sea por vergüenza o por desconocimiento. Con ello conseguimos inmensidad de logros, como la pérdida de vergüenza o la buena coordinación sobre nuestro propio cuerpo» (D.D.). «Hemos estado trabajando aspectos como seguir un ritmo, crear diferentes ritmos coordinando además nuestros movimientos y posteriormente los de toda la clase moviéndose al mismo ritmo, lo que hace que sean ejercicios muy buenos para trabajar la desinhibición» (D.S).

Algo parecido ocurre con el teatro de sombras, en el cual destacan lo educativo que resulta gracias a la desinhibición dada por la tela que impide que los alumnos sean vistos.

"Es un trabajo muy motivador y que puede ayudar a quitarse el miedo escénico, ya que, al verse la sombra, pero no a ti, no afecta tanto la timidez. Es un contenido educativo que te permite trabajar muchas cosas y que va a resultar motivador $y$ gratificante para el alumnado» (A.R.).

Grado de dificultad en la impartición de los contenidos de Expresión Corporal en el aula de Primaria (134 extractos de texto).

Con relación a la dificultad de los contenidos para impartirlos en un futuro en un aula de Primaria, los alumnos destacan la escenificación de situaciones deportivas y la desinhibición como contenidos más sencillos. Existen gran cantidad de opiniones señalando lo sencillo de algunos contenidos siempre que se tenga en cuenta una buena progresión para llevar a cabo estos, a cuál es clave para evitar problemas en algunas actividades.

"Me pareció un contenido fácil de llevar a cabo, debido a su progresión durante las actividades. Al principio nos encontramos actividades sencillas, en las cuales nos expresamos casi sin querer y poco a poco nos vamos encontrando con actividades más participativas» (D.B.). "Considero que es fácil de realizar si el docente tiene muy trabajado los contenidos y las progresiones entre los diferentes ejercicios» (D.M.). "... las actividades que realizamos nos resultaron progresivas para conseguir que las actividades finales den de sí todo lo esperado y permita trabajar correctamente el resto de la sesión» (A.R.). "Lo llevaría a cabo en clase, teniendo cuidado y trabajando con progresión para que aquellos alumnos a los que les cueste más puedan disfrutar también» (A. R.).

También destacan como algunos contenidos pueden ser más sencillos de llevar a cabo por la motivación de los alumnos de Primaria. Si los alumnos están motivados los futuros docentes ven más fácil la participación y el éxito de las actividades.

"También opino que es fácil de llevar a cabo, pues, ambientando bien la clase y escogiendo temas que gusten a los alumnos, todos van a querer participar sin ningún tipo de problema» (D.D.). "[...]crear un clima de aula favorable para animar a todos los alumnos a participar y sentirse libres para expresar (siguiendo unas directrices que marque el docente)» (A.S.).

En cuanto a la dificultad, en algunos contenidos como, la danza, para algunos alumnos esta puede ser diferente según el género. Los futuros maestros creen que los chicos tendrán más reticencias para algunos contenidos de Expresión Corporal. Esta diferencia de género es habitual dentro de la Expresión Corporal y los comentarios extraídos confirman como ese pensamiento todavía es habitual en los futuros docentes.

"[...] aunque es posible que a los chicos les cueste un poco más» (A.R.). "[...] es apreciable la diferencia de actitud de los niños comparada con la de las niñas las cuales suelen estar 
mucho más dispuestas y motivadas con los bailes por lo que se implican más a la hora de realizar las actividades» (D.S.). "Considero que es un contenido algo complicado de trabajar con los alumnos, en especial con los chicos, ya que les suele parecer un poco aburrido y dependiendo de sus habilidades rítmicas, uno puede sentirse más o menos cómodo» (M.A.).

En otros contenidos, como en el teatro de sombras, para algunos futuros docentes la dificultad puede venir por la necesidad de material e instalaciones específicas para poder llevarlo a cabo.Y son muchos los que condicionan el éxito a la posibilidad de tener medios adecuados.

"[...] pero en mi opinión difícil de llevar al aula, ya que necesitamos de unas condiciones concretas para poder llevarlo a cabo. Una sala adecuada, una tela concreta y un foco potente» (A.I.). "[...] me parece que cuenta con un gran inconveniente añadido que nos obstaculiza su realización en el área de EF. Normalmente la EF se realiza en los gimnasios o polideportivos del colegio, por lo que no se destinan aulas acondicionadas para llevar a cabo este tipo de sesiones. Considero es de vital importancia contar con un espacio adecuado para llevar a cabo el teatro de sombras, además de buenos materiales (foco de luz, tela traslúcida de calidad con dimensiones óptimas...) ya que si no la U.D. que diseñemos no será exitosa» (A.S.) «En mi opinión difícil de llevar al aula, ya que necesitamos de unas condiciones concretas para poder llevarlo a cabo. Una sala adecuada, una tela concreta y un foco potente. Pero si por suerte en el colegio se dispone de estos materiales es una actividad muy buena para llevar al aula» (D.S.).

Experiencias positivas generadas por los contenidos de EXpresión Corporal (106 extractos de texto).

La mayoría de los alumnos de magisterio comentan que los contenidos les gustaron, si bien el contenido que más comentarios positivos tiene es el teatro de sombras. En este los alumnos destacan lo novedoso que es y que no lo habían trabajado anteriormente.

"Sinceramente, me encantó. Creo que es una de las dinámicas más poco desarrolladas en las aulas» (D.D.). "Me ha gustado mucho porque no lo había practicado nunca.» (D.M.). "[...] es la mejor con diferencia, no sé cómo expresar lo interesante y divertida que puede llegar a ser» (D.B.). "Sin duda alguna fue la que más me gustó, las risas y la diversión estaban aseguradas» (J.G.).

Por otro lado, existen algunos comentarios donde los alumnos de grado expresan no haberse divertido tanto. Estos comentarios vienen referidos fundamentalmente a la clase de dramatización cuando les tocó hacer de espectadores donde los alumnos preferían actuar.

"Personalmente, se me hacía algo pesado la espera hasta que te tocaba participar» (D.M.). «Fue una sesión un poco lenta ya que estuvimos un tiempo sentados» (J.G.).

Por otro lado, en la danza los alumnos admiten que su relación y su experiencia previa con el baile, influye en lo poco o lo mucho que le gustó el contenido. Algunos de los cuales admiten que no se sienten cómodos con este.

"Fue otro de los días para los que no llevaba mucho ánimo, pues bailar así de primeras ante mi clase, no es que me hiciera especial ilusión» (D.D.). "La sesión fue entretenida pero el baile y yo no somos muy amigos. Me cuesta mucho soltarme en ese aspecto» (J.G.). "[...] baile fue una de mis favoritas y me gustó mucho ya que soy muy bailarín y danzante» (J.B.).

Cabe destacar en los comentarios extraídos como el hecho de que los contenidos de Expresión Corporal les hayan gustado en ocasiones está relacionado con la sensación de libertad, de no sentirse cohibidos y ser ellos mismos.

«Salí muy contento de la clase, me gustó mucho [...] pudimos improvisar y ser nosotros mismos» (D.D.). "[...] adquiere gran libertad y por ello me ha gustado mucho y me ha parecido muy entretenida» (M.A.). "Me gustaron bastante porque puedes ser tú mismo y mostrarte tal y como eres» (D.D.).

\section{Discusión}

La gran mayoría de alumnos del Grado de Educación Primaria Mención de Educación Física destacan el valor educativo de los contenidos de Expresión Corporal siendo los juegos de desinhibición el contenido más valorado y sencillo de impartir para los futuros maestros, no observando excesiva dificultad para impartir la mayoría de los contenidos de Expresión Corporal más allá de la motivación de los alumnos de Primaria o la necesidad de material o instalaciones. Por último, se reportan experiencias positivas tras haber vivenciado estos fundamentalmente en contenidos novedosos como el teatro de sombras.

Con relación a la primera categoría que trataba sobre los contenidos educativos en Expresión Corporal es destacable la importancia que los futuros docentes dan a los juegos de desinhibición tanto en el cuestionario como en los diarios. Los alumnos destacan la importancia de este fundamentalmente para evitar bloqueos y mejorar la confianza de los alumnos de Primaria. En el aula de Expresión Corporal se suceden acciones como observar, ser observado, tocar y ser tocado que podrían desencadenar en bloqueos y restricciones que disminuyan en el alumno su exploración y creatividad motriz (Canales-Lacruz, Táboas-Pais \& Rey-Cao, 2013), por lo que 
el trabajo de desinhibición podría condicionar en gran medida el éxito o el fracaso de una clase de Expresión Corporal. Debido a esto, los futuros docentes ven necesario trabajar los juegos de desinhibición en otros contenidos más relacionados con disciplinas artísticas (teatro, danza, ...) así, proporcionarían espacios de seguridad que posibiliten procesos exploratorios (Meirieu, 1988). Por otro lado, los futuros maestros destacan recursos educativos de otros contenidos, como puede ser la música en la danza o el trabajo detrás de la tela en el teatro de sombras, en ambos se subraya su poder desinhibitorio.

En cuanto al contenido peor valorado en esta primera categoría en el cuestionario, encontramos la escenificación de situaciones deportivas, contenido poco habitual en Expresión Corporal. Sin embargo, tras el análisis de los diarios, no existe ningún comentario que mencione algún aspecto poco educativo de este ni de ningún contenido realizado, sino que, al contrario, en todos los contenidos se reflexiona sobre sus posibilidades educativas. En general se valoran estos como bastante educativos. Estos resultados están en línea con los obtenidos por Ares y López (2012) los cuales, tras preguntar a los alumnos del Grado de Ciencias del Deporte sobre los contenidos de Expresión Corporal, encontraron como estos valoraban positivamente los contenidos propuestos considerándolos relevantes para su formación y su futuro profesional.

Con respecto a la segunda categoría sobre la dificultad de impartir contenidos de Expresión Corporal, los futuros maestros no observan los contenidos excesivamente difíciles, destacando la escenificación de situaciones deportivas y la desinhibición como los más sencillos, por lo que estos podrían ser un buen primer paso para impartir en el aula de Primaria por profesores con poca experiencia. A pesar de que los alumnos de Magisterio no ven los contenidos excesivamente difíciles a la hora de impartirlos, señalan diferentes aspectos claves relacionados con las tareas, el alumnado y el material que pueden aumentar su dificultad.

En cuanto a las actividades o tareas, los futuros docentes destacan la necesidad de realización de una buena progresión de actividades. Prat y Brunicardi (2012) tratan este aspecto a la hora de hablar de las dificultades del docente para impartir Expresión Corporal, comentan como muchos profesores tienen dudas sobre cómo realizar esta, lo cual enfatiza la importancia de una buena formación para evitar problemas más adelante. Con respecto al alumnado de Primaria, los futuros maestros destacan la necesidad de motivación de los discentes. En esta línea Archilla Prat (2013), indica como la falta de motivación o la pasividad del alumnado podría generar en el docente inseguridad. Otro aspecto que señalan los futuros maestros, relacionado con el alumnado y que podría aumentar o disminuir la dificultad para impartir contenidos, es la diferencia de género, la cual se destaca fundamentalmente en los comentarios de los diarios tras la sesión de danza. Los futuros maestros reflexionan sobre el posible rechazo en los niños y motivación en las niñas. Son varios los autores (Rodríguez et al., 2013; García \& Gutiérrez, 2002; Lluch et al., 2011) que señalan como el género es un aspecto para tratar habitualmente en la impartición de contenidos de Expresión Corporal. Por último, en cuanto al material, los futuros docentes señalan la posibilidad de contar con instalaciones y material como un aspecto necesario para impartir correctamente algunos contenidos. En este sentido, el contenido de teatro de sombras es valorado como más difícil en el cuestionario y en los diarios de sesión, son muchos los comentarios que indican la dificultad de llevar a cabo este por el material e instalación específica que requiere.

En relación con la última categoría que trata sobre las experiencias positivas generadas por los contenidos de Expresión Corporal, los futuros docentes valoran por encima de los demás, en el cuestionario, el teatro de sombras. En los diarios de sesión algunos alumnos de magisterio argumentan que les ha gustado por encima del resto porque es un contenido novedoso y que no habían trabajado anteriormente. García y Gutiérrez (2002) explican como actualmente ha habido un excesivo crecimiento de contenidos de EC siendo muchos de estos productos de modas. El incluir nuevos contenidos puede sin duda hacer que los alumnos se motiven más al trabajar contenidos novedosos, sin embargo, no hay que olvidar, tal y como nos dicen estos autores, la importancia de reflexionar sobre los objetivos educativos de estos. En esta investigación el teatro de sombras no solo se observa como un contenido novedoso, sino también educativo.

En general los alumnos reportan experiencias positivas, destacando la sensación de libertad en el trabajo de Expresión Corporal. Esta puede venir por la posibilidad de imaginar produciendo lo que Erickson (2004) denomina una dialéctica que busca la libertad de la persona y su unión con el grupo. Por otro lado, no se observan apenas comentarios negativos sobre contenidos concretos, sin embargo, a los alumnos de Grado no les gusta realizar el papel de espectador, lo cual indica que prefieren actuar. Por último, a la hora de trabajar la 
danza muchos señalan como las experiencias previas les condiciona. Prat y Brunicardi (2012) señalan que los profesores de EF a la hora de impartir contenidos de Expresión Corporal, son influidos por sus actividades de ocio personal y que la gran mayoría apenas han tenido contacto con la Expresión Corporal fuera de la Universidad. Por tanto, es especialmente importante aportar experiencias positivas para cambiar conceptos preconstruidos de los alumnos de Magisterio y así el día de mañana puedan impartir estos contenidos en Primaria.

\section{Conclusiones}

Con relación al objetivo principal del estudio se concluye que, para esta muestra, los contenidos de Expresión Corporal evaluados son en general educativos, sin un alto nivel de dificultad y generan experiencias positivas en los futuros maestros y maestras que los han trabajado.

Respecto al primer objetivo específico los futuros maestros y maestras, destacan la desinhibición como el contenido más educativo capaz de evitar bloqueos y mejorar la confianza del alumno, lo cual ayudará en gran medida al éxito en el trabajo de Expresión Corporal. Con relación al segundo objetivo específico, el alumnado no encuentra los contenidos especialmente difíciles, sin embargo, señalan la desinhibición y la escenificación de situaciones deportivas como los contenidos más sencillos. Se destaca la progresión en el nivel de dificultad de las actividades, los materiales e instalaciones necesarios, la motivación y el género del alumnado como aspectos que pueden aumentar la dificultad en la impartición de los contenidos de Expresión Corporal. Por último, en cuanto el tercer objetivo específico, el teatro de sombras es el contenido que ha generado más experiencias positivas. El alumnado destaca que el papel de espectador y las experiencias previas podrían influir negativamente en la percepción de los contenidos.

Se considera el artículo de interés para todos aquellos profesores de EF que quieran impartir contenidos de Expresión Corporal, ya que les orientará en la elección de aquellos más adecuados. También es relevante para el ámbito de la Expresión Corporal a la hora de avanzar en la delimitación de sus contenidos en la Educación Primaria y en los aspectos que condicionan el éxito o el fracaso de estos.

\section{Referencias}

Aijón, M. V. (2012). La expresión corporal, un camino tortuoso. EmásF: revista digital de Educación Física (14), 9-26.

Archilla Prat, M. T. (2013). Dificultades del profesorado de Educación Física con los contenidos de expresión corporal en secundaria (Tesis doctoral). Universidad de Valladolid. Segovia.

Ares, J. G., \& López, J. C. (2012). Relevancia de los contenidos de expresión corporal por parte de los alumnos de grado en Ciencias del Deporte. EmásF: revista digital de Educación Física (14), 106-121.

Arévalo, C. G. (2004). El deporte, una potencial herramienta formativa. Apunts. Educación Física y deportes, 3(77), 97-101.

Arias, J. R., Fernández, B. \& San Emeterio, C. (2020). Construcción y validación de un instrumento para la medida de las actitudes hacia la Expresión Corporal. Retos: nuevas tendencias en Educación Física , deporte y recreación, (38), 443-451.

Arias, J.R, Fernández, B. \& Valdés R., (2021). Actitudes hacia la Expresión Corporal en el ámbito de la asignatura de Educación Física : Un estudio con alumnado de Educación Secundaria Obligatoria (Attitudes towards Body Expression in the field of Physical Education subject: A study with Compulsory Se. Retos, (41), 596-608.

Arizcuren, E. (2013). La exploración, expresión y comunicación percibida por el alumnado universitario ante situaciones motrices Artístico-expresivas. Trabajo fin de máster. Zaragoza: Universidad de Zaragoza. Recuperado de: https: / / zaguan.unizar.es/record/13241 / files/TAZ-TFM-2013-1289.pdf

Bores-García, D., Hortigüela-Alcalá, D., HernandoGarijo, A., \& González-Calvo, G. (2021). Analysis of student motivation towards body expression through the use of formative and share assessment (Análisis de la motivación del alumnado hacia la expresión corporal a través del uso de la evaluación formativa y compartida). Retos, (40), 198-208.

Canales, I., Táboas-Pais, M. I. \& Rey-Cao, A (2010). Desinhibición, espontaneidad y codificación percibida por el alumnado de expresión corporal. Movimento, 19 (4), 119-140.

Canales-Lacruz, I., \& Arizcuren-Blasco, E. (2019). Feelings and opinions of Primary School Teacher Trainess towards corporal expressivity, spontaneity, and disinhibition. Research in dance education, 20, 1-16. doi.org/10.1080/14647893.2019.1572732

Canales-Lacruz, I. \& Corral-Abós, A. (2021). Interacción visual y lógica interna en las tareas de Expresión Corporal. Retos: nuevas tendencias en Educación Física , 
deporte y recreación, 40, 224-230.

Elliott, J. (2000). El cambio educativo desde la investigaciónacción. Madrid: Ediciones Morata.

Erickson, D. (2004). Creative dance and basketball. Teaching Elementary Physical Education, 15(2), 38-40. García, L., \& Gutiérrez, D. (2002). Análisis y estructuración de los contenidos de expresión corporal. Revistas de la Universidad, 2, 1-25.

Garzón, P. C., Fernández, M. D., Sánchez, P.T., \& Gross, M. G. (2002). Actividad físico-deportiva en escolares adolescentes. Retos: nuevas tendencias en Educación Física, deporte y recreación (3), 5-12.

Gil, J. (2016). Los contenidos de la Expresión Corporal en el Título de Grado en Ciencias del Deporte. [Tesis Doctoral]. Madrid: Universidad Politécnica de Madrid.

Köpsén, S. (2014). How Vocational Teachers Describe Their Vocational Teacher Identity. Journal of Vocational Education and Training, 66(2), 194-211.

Ley Orgánica 8/2013, de 9 de diciembre, para la mejora de la calidad educativa. Boletín Oficial del Estado. 295, 97858-97921. Recuperado de https:// www.boe.es/eli/es/lo/2013/12/09/8/dof/spa/ pdf

Llopis, A., \& García, M.E. (2012). Danza: experiencias prácticas de los estudiantes de la diplomatura de Educación Física y de la licenciatura de ciencias de la actividad Física y el deporte. Tándem, 39, 22-36

LLuch, Á. C., Urzúa, M. F., León-Prados, J. A., \& Sánchez, I. G. (2011). Un análisis DAFO sobre expresión corporal desde la perspectiva de la Educación Física actual. EmásF: revista digital de Educación Física (11), 20-28.

Meirieu, P., \& Olcina, E. (1998). Frankenstein educador. Barcelona: Laertes.

Megías, M. E. P. (2020). Pensar el cuerpo: de la expresión corporal a la conciencia» expresivo corporal», un camino creativo narrativo en la formación inicial del profesorado. Retos: nuevas tendencias en Educación Física , deporte y recreación, (37), 643-651.

Min-Hau, C., \& Phillips, D. A. (2002). The relationship between attitude toward physical education and leisure time exercise in high school students. Physical Educator, 59(3), 126.

Moreno, J. A., \& Hellín, M. (2007). El interés del alumnado de Educación Secundaria Obligatoria hacia la Educación Física . Revista electrónica de investigación educativa, 9(2), 1-20.

Oliver-Hoyo, M., \& Allen, D. (2006). The Use of Triangulation Methods in Qualitative Educational Research. Journal of College Science Teaching, 35(4),
42-47.

Prat, M. T. A., \& Brunicardi, D. P. (2012). Dificultades del profesorado de EF con las actividades de expresión corporal en secundaria. EmásF: revista digital de Educación Física (14), 176-190.

Real Decreto 126/2014, de 28 de febrero, por el que se establece el currículo básico de la Educación Primaria. Boletín Oficial del Estado, 52, 19349-19420. Recuperado de https://www.boe.es/boe/dias/2014/ 03/01/pdfs/BOE-A-2014-2222.pdf

Rodríguez, J. R., Robles, M. T. A., Viera, E. C., Fuentes-Guerra, F. J. G., \& Rodríguez, A. R. (2013). Factores que condicionan la presencia de la expresión corporal en la enseñanza secundaria según el profesorado de Educación Física . Retos. Nuevas tendencias en Educación Física , Deporte y Recreación (24), 171 175.

Sallis, J. F., Prochaska, J. J., \& Taylor, W. C. (2000). A review of correlates of physical activity of children and adolescents. Medicine \& science in sports \& exercise, 32(5), 963-975.

Saldaña, J. (2009), The Coding Manual for Qualitative Researchers, Thousand Oaks, CA: Sage.

Sánchez, G., \& López, M. (2019). Análisis de los contenidos de Expresión Corporal impartidos en la formación inicial de los docentes de Primaria. Educación XX1, 22(1).

Sánchez, M. C. (2015). La dicotomía cualitativo-cuantitativo: posibilidades de integración y diseños mixtos. Campo abierto. Revista de Educación, 11-30.

Torrents, C., Mateu, M., Planas, A., \& Dinusôva, M. (2011). Posibilidades de las tareas de expresión corporal para suscitar emociones en el alumnado. Revista de Psicología del deporte, 20(2), 401-412.

Tortorella, G. L., Viana, S., \& Fettermann, D. (2015). Learning cycles and focus groups: A complementary approach to the A3 thinking methodology. The Learning Organization. 22 (4), 229-240.

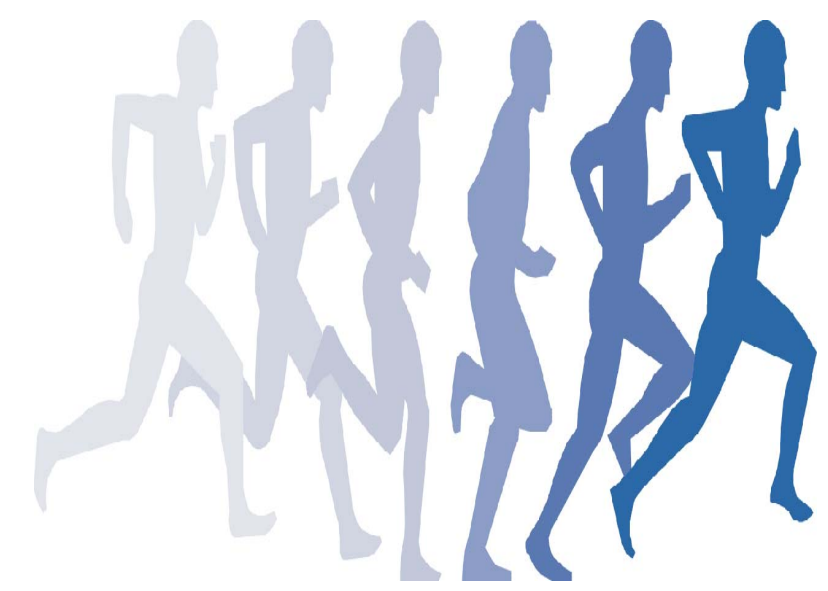

